\title{
Arteria subclavia aberrante izquierda, hallazgo infrecuente
}

\author{
Juan Francisco Allamand T. ${ }^{*}$, Francisco Carriel A. ${ }^{2}$, Verynica Hamilton M. $^{3}$, Mathas Molina $V^{4}$, Gonzalo Pumarino M5.
}

1. Cirujano, Servicio de Emergencias Adulto, Hospital Padre Hurtado. Santiago, Chile.

2. Residente de Cirugía, Facultad de Medicina Clínica Alemana-Universidad del Desarrollo. Santiago, Chile.

3. Interna de Medicina, Facultad de Medicina Clínica Alemana-Universidad del Desarrollo. Santiago, Chile.

4. Radiólogo, Servicio de Imagenología, Hospital Padre Hurtado. Santiago, Chile.

5. Cirujano, Servicio de Cirugía, Hospital Padre Hurtado. Santiago, Chile.

\section{Aberrant left subclavian artery, an uncommon variation}

Resumen: Objetivo: La arteria subclavia aberrante (ASA) o arteria lusoria, es una de las malformaciones más comunes del arco aórtico; en especial su variante derecha. Por otro lado, la arteria subclavia aberrante izquierda es más rara. Presentamos un caso infrecuente de un paciente con síndrome deleción $22 q 11.2$ y ASA izquierda con síntomas en extremidad superior izquierda. La ASA izquierda es una condición muy poco frecuente y hay escasa información sobre su tratamiento quirúrgico. La mayoría de las veces, el ASA es asintomática; especialmente en adultos. Si presenta clínica, sus síntomas son disfagia, tos, disnea, claudicación de la extremidad superior. La presencia de sintomatología es una indicación quirúrgica. El tratamiento quirúrgico de una ASA es discutido. Éste va a depender de la anatomía, comorbilidades y experiencia del cirujano.

Palabras clave: Arteria subclavia aberrante izquierda, Arteria lusoria, Cirugía endovascular, Cirugía híbrida.

Abstract: Objective: The Aberrant Right Subclavian Artery (ARSA) or Lusoria Artery is one of the most common aortic arch malformations. Aberrant Left Subclavian Artery (ALSA) on the other hand, is a much rarer condition. We present an uncommon case of ALSA in a patient with 22q11.2 deletion syndrome with upper limb symptoms and review the treatment options. ALSA is an exceedingly rare condition and information on its surgical treatment is scarce. In most cases the presence of an ALSA is asymptomatic, especially in adults. Some of the most typical symptoms are dysphagia (dysphagia lusoria), cough, dyspnea, claudication of the upper limb extremity. When symptomatic, the patient has indication of surgery. The treatment of an ALSA is still debatable and it depends on the anatomy, comorbidities and surgeon's experience.

Key words: Aberrant left subclavian artery, Hybrid procedure, Lusoria artery.

Allamand JF, et al. Arteria subclavia aberrante izquierda, hallazgo infrecuente. Rev Chil Radiol 2019; 25(2): 71-74. *Correo electrónico: Juan Francisco Allamand T. / juanfcoallamand@gmail.com.

Trabajo enviado el 13 de mayo de 2019. Aceptado para publicación el 06 de junio de 2019.

\section{Introducción}

La arteria subclavia aberrante (ASA) o arteria lusoria, es una de las malformaciones vasculares congénitas del arco aórtico más comunes; siendo la ASA derecha su variante más frecuente, con un $0,4-2,3 \%$ de prevalencia ${ }^{(1)}$. La ASA izquierda, por otro lado, es una condición rara con una prevalencia de 0,04-0,4\%; y hasta dos veces más frecuente en hombres de hasta el doble ${ }^{(2)}$.

El origen de esta malformación aún está en discusión, sin embargo, lo más aceptado hasta el momento es la alteración de un adecuado desarrollo embriológico del arco aórtico(2). Hasta en un $60 \%$ de las veces, la ASA se encuentra asociada a un divertículo de Kommerell en su origen. A su vez, éste puede crecer en diámetro y en ocasiones ser punto de partida de una disección ${ }^{(1,2)}$.

Sólo en un $5 \%$ de los casos esta condición es sintomática, presentándose en la población adulta con disfagia (34\%), disnea (25\%), dolor torácico (16\%), tos $(8 \%)$, claudicación de la extremidad superior correspondiente $(5 \%)$, entre otros ${ }^{(2)}$. 
Dentro de las indicaciones quirúrgicas en estos pacientes se encuentran la existencia de molestias clínicas y/o la presencia de un divertículo de Kommerell mayor a $30 \mathrm{~mm}^{(1,3)}$. Las alternativas terapéuticas incluyen la cirugía abierta, híbrida y endovascular. Se debe considerar cada caso individualmente para elegir la técnica quirúrgica según anatomía, comorbilidades y la experiencia del cirujano, aunque los estudios se inclinan hacia un manejo con cirugía endovascular/ híbrida ${ }^{(4,5)}$.

Se presenta un caso en un paciente de 25 años con ASA izquierda sintomática.

\section{Presentación del Caso}

Paciente de sexo masculino de 25 años, portador del Síndrome diGeorge (o síndrome de deleción 22q11.2), con antecedente de coartación aórtica operada en su infancia.

Refiere una historia de 2 meses de dolor intermitente y pérdida de fuerza en la extremidad superior izquierda (ESI).

Un angioTAC de tórax muestra un arco aórtico a derecha (Figura 1), con arteria subclavia aberrante izquierda retro-esofágica, sin estenosis significativa, emergiendo de la aorta descendente (Figura 2). Las arterias carótidas común y subclavia derechas se originan separadas; esta última con dilatación bulbosa en su origen (Figura 3a y 3b). La electromiografía

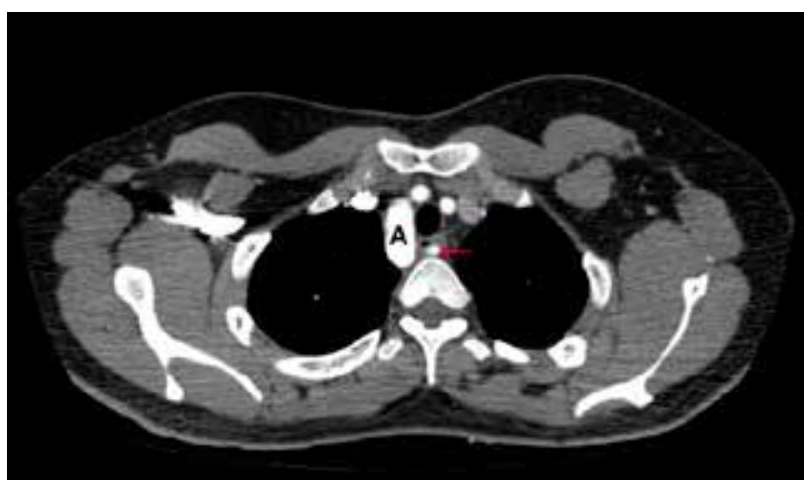

Figura 1: Arco aórtico derecho (A) y ASA retroesofágica (flecha roja).

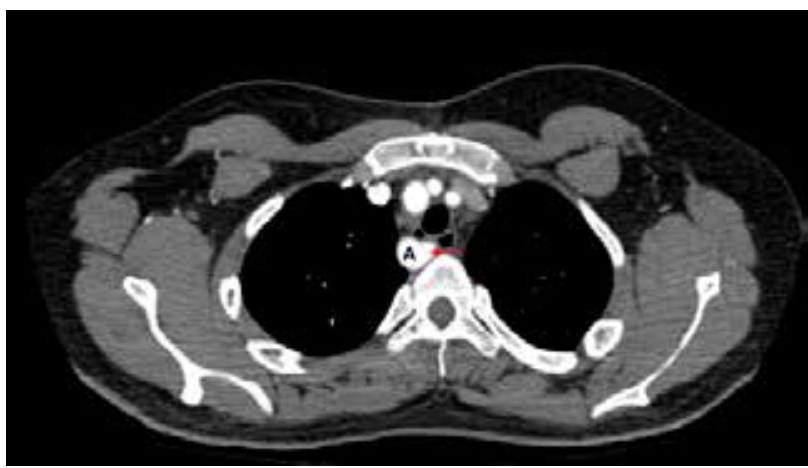

Figura 2: ASA retroesofágica emergiendo de la aorta descendente (flecha roja). de la ESI es normal. El paciente y su madre niegan disfagia, disnea, dificultad respiratoria, tos crónica u otros síntomas.

Se discute una eventual cirugía, pero los síntomas se resuelven, por lo que se decide manejo expectante.
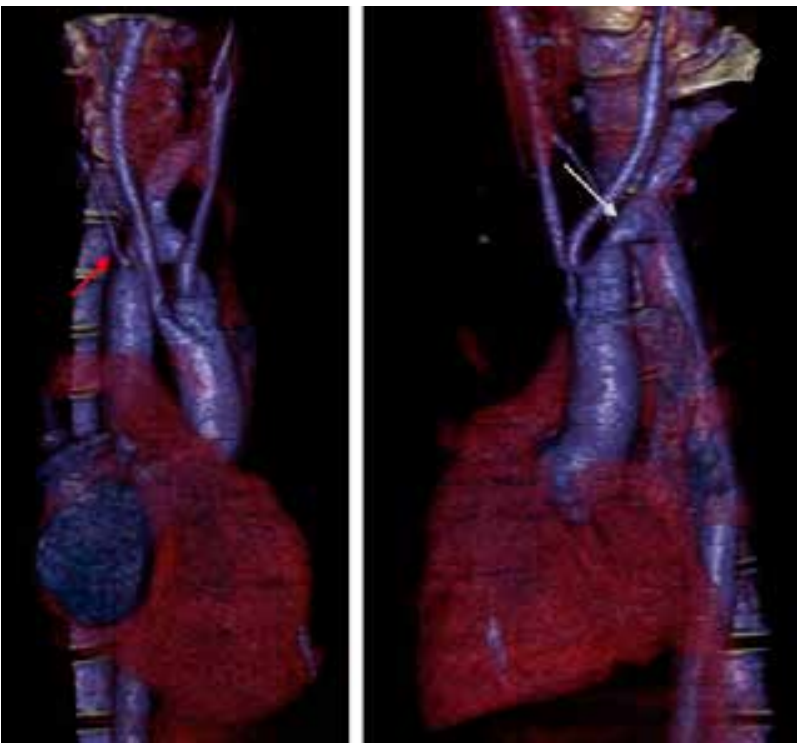

Figura 3a y 3b: Reconstrucción 3DVR. Es visible una dilatación del origen de la sublavia derecha (flecha blanca) y el curso aberrante de la subclavia izquierda (flecha roja).

\section{Discusión}

La arteria subclavia aberrante (ASA) es la anomalía congénita más frecuente del arco aórtico, pudiendo encontrarse en el 0,5-2,52\% de la población, siendo la ASA derecha más habitual, con una incidencia de entre $0,4-2,3 \%{ }^{(1,6)}$. La variante izquierda es un hallazgo infrecuente, con estudios que refieren una prevalencia desde el 0,04 al 0,5\% de la población, asociado a un arco aórtico derecho ${ }^{(2,6,7)}$. Esta anomalía se da en hombres hasta dos veces más que en las mujeres ${ }^{2}$.

En pacientes con síndromes genéticos aumenta la prevalencia de ASA, tanto derecha como izquierda, en especial en los síndromes de Down y deleción 22q11.2. Un estudio demostró que un $17 \%$ de los pacientes con síndrome de Down tienen una arteria subclavia aberrante derecha; y por otro lado demostró que hasta un $30 \%$ de los pacientes con síndrome deleción 22q11.2 presentan un arco aórtico derecho, el cual se asocia a ASA izquierda en un $47 \%$ de los $\operatorname{casos}^{(7,8)}$.

A pesar de que su origen embriológico sigue en debate, se presume que tanto la ASA derecha como la izquierda ocurren por la interrupción de los precursores embriológicos de la vasculatura en sitios incorrectos ${ }^{(2)}$. La ASA derecha ocurre cuando se interrumpe el cuarto arco proximal al eventual origen de una subclavia normal, naciendo ésta de la aorta dorsal. La ASA izquierda, en cambio, ocurre cuando degenera el cuarto arco izquierdo. Así, se genera una 
aorta a derecha y el origen de la ASA izquierda queda en la aorta descendente ${ }^{2}$. La presencia de una arteria lusoria izquierda sin un arco aórtico derecho es aún más anecdótica y hasta donde tenemos conocimiento existen sólo dos casos reportados ${ }^{(9,10)}$ (Figura 4).

Está descrito que el $60 \%$ de las veces se encuentra un aneurisma en el origen de la ASA derecha, debido a una falla en la involución de la aorta dorsal ipsilateral, lo que se conoce como divertículo de Kommerell(2). Es común también la asociación con otras malformaciones, como la existencia de un tronco carotídeo común, anomalías en el origen de la arteria vertebral o de su entrada a la espina cervical, dextrocardia, entre otros ${ }^{(7,8)}$.

En la población adulta la mayoría de los casos son asintomáticos y se diagnostica incidentalmente en el estudio de imágenes o como hallazgo en autopsias ${ }^{(6,11)}$. Cuando presenta síntomas, el más frecuente es la disfagia lusoria (34\%), presentándose en general en la cuarta y quinta década de vida. Se pueden asociar también a disnea $(25 \%)$, dolor torácico $(16 \%)$, tos crónica $(8 \%)$, claudicación de extremidades superiores (5\%) y síntomas neurológicos, siendo estos últimos más $\operatorname{raros}^{(2)}$.

Al ser la mayoría de los casos a derecha, no está aún bien documentado el cuadro clínico en presencia de una ASA izquierda, si bien suelen ser similares. En general la información proviene de reportes de casos y a estudios realizados en población pediátrica, ya que en este caso se presentan síntomas respiratorios debido a la existencia de una traqueomalacia en la infancia $^{(7)}$.

Es difícil encontrar consenso sobre el mejor trata- miento en caso de una ASA izquierda. Actualmente no existe acuerdo sobre el tratamiento en esta patología, y los reported de resultados de los pacientes tratados sólo se reducen a pequeñas series de $\operatorname{casos}^{(11)}$.

La cirugía en un paciente con ASA está indicada en pacientes sintomáticos o con dilatación aneurismática asociada para evitar complicaciones como rotura, la cual tiene una mortalidad de un $50 \%{ }^{(1)}$. Cina et al. ${ }^{(3)}$ agregan como indicación quirúrgica la presencia de un divertículo de Kommerell mayor a $30 \mathrm{~mm}$, por el riesgo de rotura o de disección aórtica. Sin embargo, aún está en discusión cómo y dónde realizar la medición de éste(2).

El objetivo del tratamiento quirírgico es resolver la compresión extrínseca de las estructuras mediastínicas (esófago principalmente), tratar el aneurisma y revasculizar la extremidad. La complejidad de la técnica a emplear dependerá principalmente de la presencia de otras variantes o hallazgos asociados (ej. un divertículo de Kommerell de gran tamaño podría obligar a tratar también el cayado, no sólo la arteria lusoria). Así, la vía clásica fue por décadas la toracotomía izquierda o derecha para el reemplazo de la aorta descendente y la reinserción de la arteria lusoria. Su morbimortalidad depende de la complejidad del caso, pero series de reemplazo de aorta torácica y revascularización del brazo destacan una mortalidad de hasta $11 \%^{(2)}$ y morbilidad entre 18 y $29 \% \%^{(1,2,12)}$.

Hoy, si no es necesario tratar la aorta o al divertículo, lo más frecuente es la confección de un puente carótido subclavio para posteriormente ligar la ASA proximal al puente. Con el advenimiento de técnicas endovasculares, es frecuente que se agregue a este

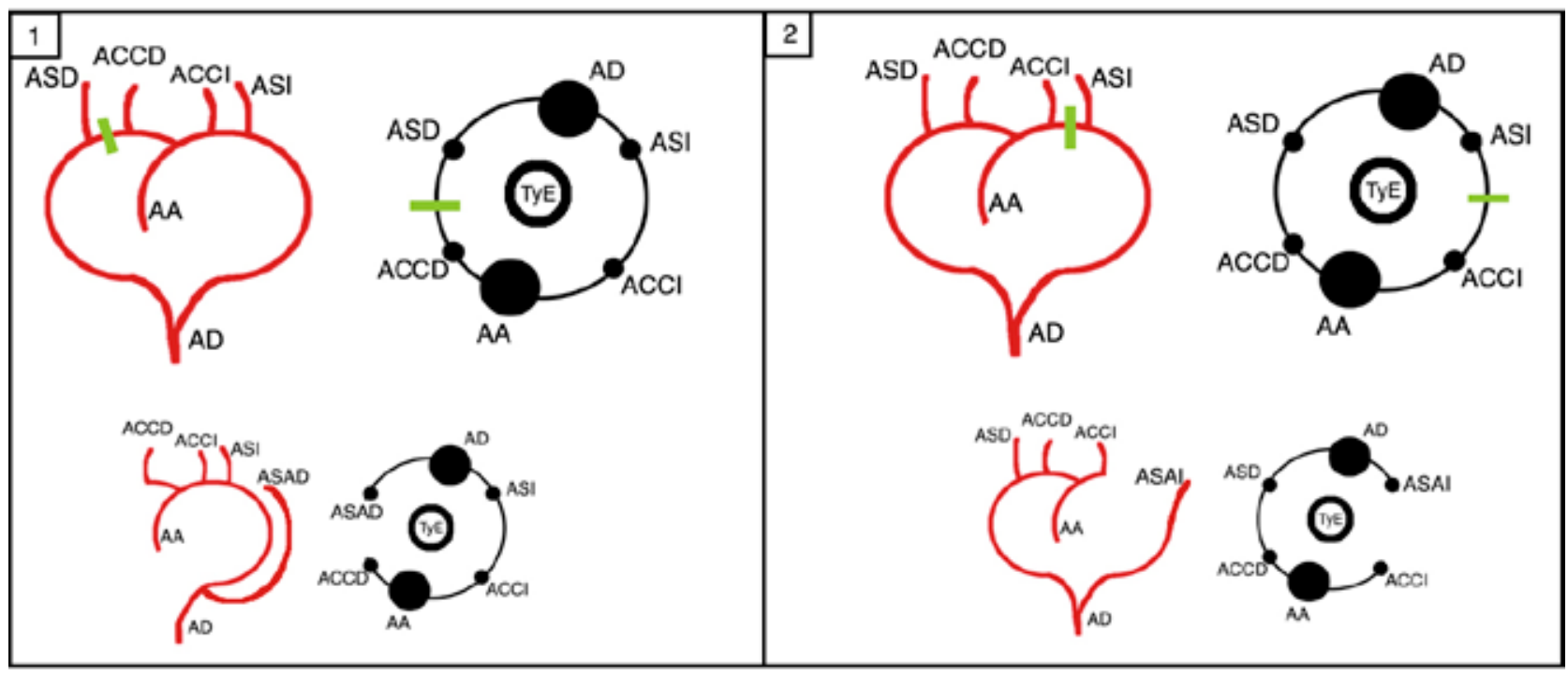

Figura 4:1: La Interrupción del cuarto arco aórtico entre la yema de la carótida derecha y la subclavia derecha provoca una ASA derecha desde la aorta dorsal. 2: La Interrupción del cuarto arco aórtico entre la yema de la carótida izquierda y la subclavia izquierda provoca un arco aórtico derecho y una $A S A$ izquierda. $A A$, aorta ascendente; $A D$, aorta dorsal; $A C C D$, carótida común derecha; $A C C l$, carótida común izquierda; $A S D$, subclavia derecha; $A S I$, subclavia izquierda; $A S A D$, subclavia aberrante derecha; ASAl, subclavia aberrante izquierda; TyE, tráquea y esófago. Modificado de Tanaka et al . 
procedimiento la embolización del remanente arterial. Para ello existen diversas opciones, siendo los dispositivos de cierre (plugs p. ej. Amplatzer) insertados desde el mismo cabo a ligar una de las más utilizadas ${ }^{(1,13)}$.

Por el contrario, para casos que requieran tratar la aorta, existen diversos reportes de manejo híbrido que incluyen la exclusión del aneurisma y el origen de la arteria lusoria con una endoprótesis aórtica más la revascularización de la arteria lusoria ${ }^{(4,13,14)}$.

Existe escasa literatura sobre los resultados quirúrgicos en manejo de ASA, sobretodo al largo plazo; siendo los resultados a corto y mediano plazo optimistas respecto a estas técnicas ${ }^{(1,4,5)}$.

\section{Conclusión}

Dentro de las variaciones anatómicas del arco aórtico, la arteria lusoria es relativamente infrecuente. Mucho menos común es la arteria lusoria izquierda, la cual puede asociarse a otras malformaciones congénitas y alteraciones como el Síndrome diGeorge.

Dependiendo de las alteraciones asociadas, es posible que el manejo requiera de maniobras de mayor complejidad, combinando técnicas híbridas que resuelvan endovascularmente la aorta y en forma abierta la revascularización de la arteria subclavia. Siendo esta una entidad de baja prevalencia es necesario un enfoque multidisciplinario para cada caso.

Conflicto de Intereses: Ninguno.

\section{Referencias}

1. Yang C, Shu C, Li M, Li Q, Kopp R. Aberrant subclavian artery pathologies and Kommerell's diverticulum: a review and analysis of published endovascular/hybrid treatment options. J Endovasc Ther 2012; 19(3): 373382.

2. Tanaka A., Milner R., Ota T. Kommerell's diverticulum in the current era: a comprehensive review. Gen Thorac Cardiovasc Surg 2015; 63(5): 245-259.

3. Cina C.S, Althani H, Pasenau J, Abouzhar L. Kommerell's diverticulum and right-sided aortic arch: A cohort study and review of the literature. J Vasc Surg 2004; 39: 131139.

4. Vucemilo I, Harlock JA, Qadura M, Guirgis M, Gowing RN, Tittley JG. Hybrid repair of symptomatic aberrant right subclavian artery and Kommerell's diverticulum. Ann Vasc Surg. 2014; 28(2): 411-420.

5. Van Bogerijen GHW., et.al. Evolution in the Management of Aberrant Subclavian Arteries and Related Kommerell Diverticulum. Ann Thorac Surg, 2015; 100(1): 47-53.

6. Polednak AP. Prevalence of the aberrant right subclavian artery reported in a published systematic review of cadaveric studies: The impact of an outlier. Clin Anat 2017; 30(8): 1024-1028.

7. Tyczyński P. et al., Left aberrant subclavian artery. Systematic study in adult patients, Int J Cardiol 2017; 240: 183-186.

8. Oswal N., Christov G., Sridharan S., Khambadkone S., Bull C., Sullivan I. Aberrant subclavian artery origin in tetralogy of Fallot with pulmonary stenosis is associated with chromosomal or genetic abnormality. Cardiol Young 2013; 24(03): 478-484.

9. Yu PJ., Balsam LB., Mussa FF., DeAnda A. Aberrant Left Subclavian Artery Associated with a Kommerell's Diverticulum and a Left-Sided Aortic Arch. J Card Surg 2012; 27(5): 607-608.

10. Komiyama M., Yasui T. Left subclavian artery originating from Kommerell diverticulum in the left aortic arch. $J$ Thorac Cardiovasc Surg 2006; 132(6): 1477.

11. Myers PO, Fasel JH, Kalangos A, Gailloud P. Arteria lusoria: developmental anatomy, clinical, radiological and surgical aspects. Ann Cardiol Angeiol 2010; 59(3): 147-154.

12. Kieffer E, Bahnini A, Koskas F. Aberrant subclavian artery: surgical treatment in thirty-three adult patients. J Vasc Surg 1994; 19(1): 100-111.

13. Jalaie H. et al. Treatment of Symptomatic Aberrant Subclavian Arteries. Eur J Vasc Endovasc Surg 2014; 48(5): 521-526.

14. Wooster M., Back M., Sutzko D., Gaeto H., Armstrong P., Shames M. A 10-Year Experience Using a Hybrid Endovascular Approach to Treat Aberrant Subclavian Arterial Aneurysms. Ann Vasc Surg 2018; 46: 60-64. 